\title{
Modelled Excitation Functions of ${ }^{112,114,116,118,120,122,124}$ Sn Isotopes for an Incident Energy Range of 10-80 MeV
}

\author{
Halim Büyükuslu* \\ Giresun University, Espiye Vocational School, Department of Medical Services and Techniques, 0 (454) 3101430 , \\ halimbuyukuslu@gmail.com \\ * Corresponding Author
}

Received: 27 March 2017

Accepted: 14 December 2017

DOI: $10.18466 /$ cbayarfbe. 370373

\begin{abstract}
The data of the cross-section is required for understanding nuclear reaction mechanism, developing and testing validity of available nuclear reaction models as much as it has been used in the applied fields of nuclear physics. Reliable experimental data is importance of comparison with theoretical nuclear model calculations for testing predictive ability. In the event that experimental data failure appearance, theoretical models are filling this gab for supplying with calculated data. Because of these reasons, both of experimental and theoretical studies need of each other. For a long time, theoretically studies have been carried out for proton total reaction cross section calculation. At the end of these studies, several analytical equations have been proposed by their authors. Comparison of the suggested equations and rearrangement for different value of parameters are enormously important for reaching successfully results. In this study, proton total reaction cross sections have been calculated for $112,114,116,118,120,122,124 \mathrm{Sn}$ isotopes using proton-nucleus interaction analytic equation that was proposed by M.A. Alvi. Also, optical model calculations carried out for same reactions by TALYS code. Excitation functions have been plotted with collected experimental data up to $80 \mathrm{MeV}$ proton incident energy. Coefficient comparisons have been made via determined excitation function curves. The obtained results have been discussed by way of the excitation function graphics and compared with the available experimental data. Satisfactory agreements have been seen between calculated data and its measured equivalents. A validation of used theoretical model has been confirmed and tested via obtained results for these $\mathrm{Sn}$ isotopes and these energy ranges.
\end{abstract}

Keywords: Excitation functions, Glauber Model, Proton Induced Reactions, Talys Code Calculations, Total Reaction Cross Section

\section{Introduction}

Proton induced reactions offer essential tool for the nuclear structure researches to determine the nuclear size, shape, interaction potential nuclei. Especially high energetic proton interactions with the nuclei able to clarifies nucleonnucleon nuclear dynamics. Such studies are also expected to provide significant insight into the reaction mechanisms dominant in different energy regimes.

Highly numbers of experimental cross section data are need to nuclear science applications. Especially, the production of medical radionuclides requires the evaluation of both experimental and calculated cross sections. Optimum neutron production method is the basic aim for reactor technology. Hundreds number of target isotopes are hit by light charged particle like proton for producing huge neutron fluxes. Neutron beams are used wide range of area such as reactor physics, solid-state physics and energy production.
Radiation shielding, space sciences, nuclear waste treatment, accelerator-driven subcritical reactors and etc are major applied technologies base on neutron emission reactions.

Theoretical nuclear models that have successful predictive ability are efficient solution for measuring problems such as radioactive half time, economic constriction and physical handicaps. When there are inadequate experimental cross section data, theoretical models can supplement this deficiency. Reliable models provide to gain about economic and time for researchers. Predictive power is one of importance criterion for nuclear model validity. More realistic models be able to contribute to better neutron induced application of nuclear technology.

In this work, results of total reaction cross section calculations are presented for the following target $\mathrm{Sn}$ isotopes: 
${ }^{112} \mathrm{Sn},{ }^{114} \mathrm{Sn},{ }^{116} \mathrm{Sn},{ }^{118} \mathrm{Sn},{ }^{120} \mathrm{Sn},{ }^{122} \mathrm{Sn}$, and ${ }^{124} \mathrm{Sn}$. M.A. Alvi [1] proton-nucleus analytic relation has been used for total reaction cross section calculation at the proton incident energy range of 10-80 MeV. Also TALYS code calculations for mentioned reactions have been added for testing results' validation. Comparison have been carried out between experimental data taken from EXFOR database [2] and determined cross section values.

\section{Material and Method}

High-energy collisions were in sighted with microscopic nucleon-nucleon interaction approach by the Glauber model [3]. More of total cross section studies based on this reaction theory. Alvi [1] presented an analytic equation for proton-nucleus total reaction cross section using the Helm model nuclear form factor and working within the framework of the Coulomb modified Glauber model. Also proton total cross sections calculations have been determined for some target nuclei at incident energy range of 10-860 MeV $[1,4]$.

A semi-phenomenological closed expression for proton total reaction cross section $\sigma_{R}$ has proposed by Alvi, as

$$
\begin{aligned}
\sigma_{R}= & 2 \pi\left(c_{1} N^{2 / 3}+\sigma^{2}+\beta_{N N}^{2}\right)\left(1-\frac{1.44 Z(A+1)}{A E_{L} R_{i n t}^{*}}\right) \\
& \times \ln \left[c_{2} A /\left(2\left(c_{1} N^{2 / 3}+\sigma^{2}+\beta_{N N}^{2}\right)\right]\right.
\end{aligned}
$$

where

$$
\begin{aligned}
R_{\text {int }}^{*} & =\sqrt{2\left(c_{1} N^{2 / 3}+\sigma^{2}+\beta_{N N}^{2}\right)} \\
& \times \sqrt{\ln \left[c_{2} \xi A /\left(2\left(\left(c_{1} N^{2 / 3}+\sigma^{2}+\beta_{N N}^{2}\right)\right)\right]\right.}
\end{aligned}
$$

with $\xi=\left(e^{\gamma} \ln 2\right)$

In Equation. (2.1), $c_{1}$ and $c_{2}\left(=e^{\gamma} c_{0} \sigma_{N N} / \pi\right)$ are two parameters, $E_{L}$ is the lab energy in $\mathrm{MeV}, N$ and $A$ are the neutron and mass numbers of the target nucleus, respectively.

The default optical model potentials (OMP) used in TALYS [5,6] are the local and global parameterisations of Koning and Delaroche [7]. Default parameter's values have been used in these calculations. Detailed description about the theory and parameterisations of code can be seen from ref $[5,6]$.

The phenomenological OMP for nucleon-nucleus scattering, $U$, is defined as:

$$
\begin{aligned}
& U(r, E)=-V_{V}(r, E)-i W_{V}(r, E)-i W_{D}(r, E) \\
& +V_{S O}(r, E) .1 . \sigma-i W_{S O}(r, E) \cdot 1 \cdot \sigma+V_{C}(r)
\end{aligned}
$$

where $V_{V, S O}$ and $W_{V, D, S O}$ are the real and imaginary components of the volume-central $(V)$, surface central $(D)$ and spin-orbit $(S O)$ potentials, respectively. $E$ is the lab energy of the incident particle in $\mathrm{MeV}$.

\section{Results}

Proton total reaction cross-sections were calculated by using Equation (2.1) and optical model by TALYS code at the proton incident energy range of $10-80 \mathrm{MeV}$ for Sn target isotopes. Equation (2.1) and Equation (2.2) include several parameters and quantities such as Helm [8] model form factor parameters $\left(\propto_{0}^{2}, c_{0}, R\right.$ and $\left.\sigma\right)$ and $N N$ scattering amplitude parameters $\left(\beta_{N N}^{2}, \alpha_{N N}, \sigma_{N N}\right)$. Well-matched parameters have been chosen for carrying out best agreement results. Charagi's [9] parameterization have been preferred for valuating $\sigma_{p p}$ and $\sigma_{p n}$ quantities and Ahmad's [10] for $\alpha_{p p}$ and $\alpha_{p p}$. Parameters, their relations/values are given with their references in Table 1.

Table 1. The parameters, relations/values and their Refs.

\begin{tabular}{|c|c|c|}
\hline Parameter & Relation/Value & Ref \\
\hline$\beta_{N N}^{2}$ & 0.423 & {$[9]$} \\
\hline & $\frac{1+\alpha_{N N}^{2}}{16 \pi} \sigma_{N N}$ & {$[11]$} \\
\hline$\propto_{0}^{2}$ & 0.115 & \\
\hline$c_{0}$ & 1.035 & \\
\hline
\end{tabular}

$\sigma_{R}$ proton total cross section calculations have been performed for different references values of $\beta_{N N}^{2}$. The value $0.423 \mathrm{fm}^{2}$ was reported by Charagi and Gupta [9]. The other relation that is shown in Table 1 was suggested by Horiuchi et al. [11]. 0.4 value was preferred for energy independent free coefficient $c_{1}$ by M.A. Alvi. In order to get best correlation with the experimental data, $c_{1}$ has been chosen 0.36 value in Figures 1-3 and 0.4 value in Figures 4-7. $c_{2}$ has been determined from relation of $c_{2}(=$ $\left.e^{\gamma} c_{0} \sigma_{N N} / \pi\right)$. All of theoretical calculated cross sections have been contrasted with the each other and measured values collected from EXFOR.

Owing to experimental excitation functions that were taken from literature have continuous up to $75 \mathrm{MeV}$ proton induced energy, theoretical calculations have been carried out up to about $80 \mathrm{MeV}$ incident energy. The comparisons between the theoretical and experimental cross section are shown in Figures 1-7. 


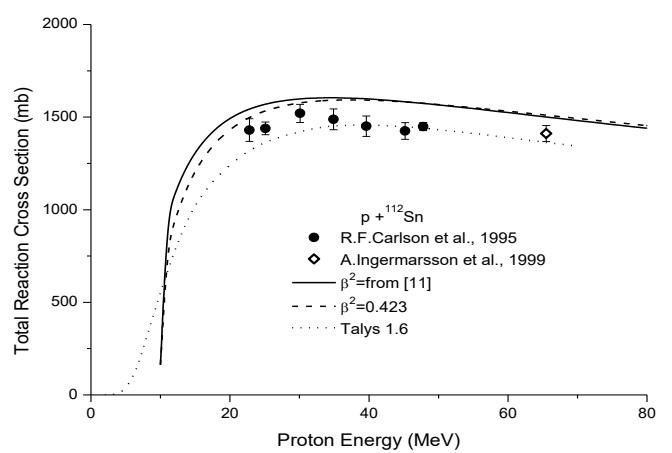

Figure 1. Calculated excitation function curves and experimental values for ${ }^{112} \mathrm{Sn}$ target nucleus. Experimental values were taken from Ref. [2]

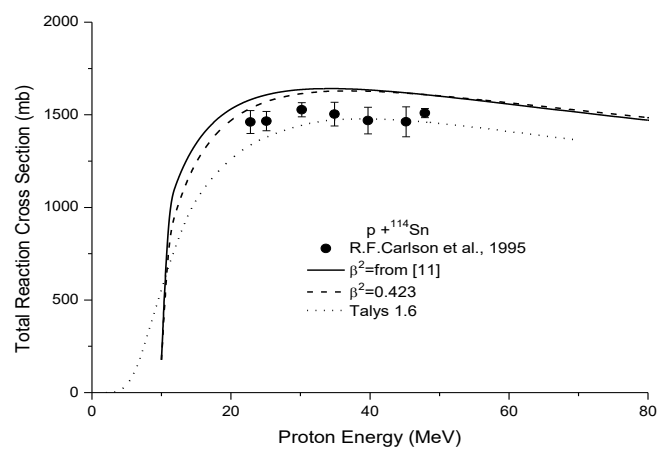

Figure 2. Calculated excitation function curves and experimental values for ${ }^{114} \mathrm{Sn}$ target nucleus. Experimental values were taken from Ref. [2]

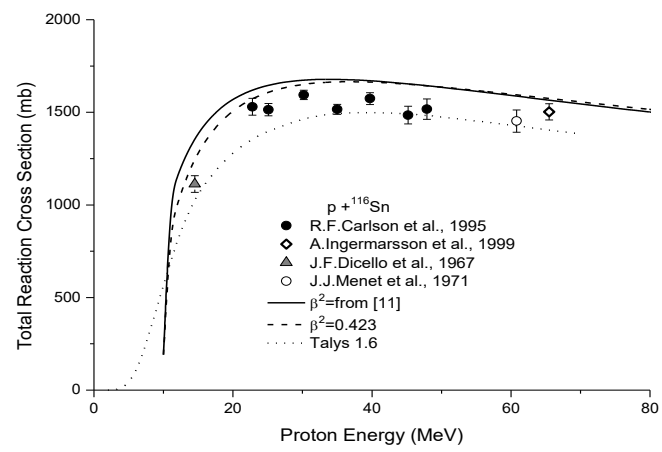

Figure 3. Calculated excitation function curves and experimental values for ${ }^{116} \mathrm{Sn}$ target nucleus. Experimental values were taken from Ref. [2]

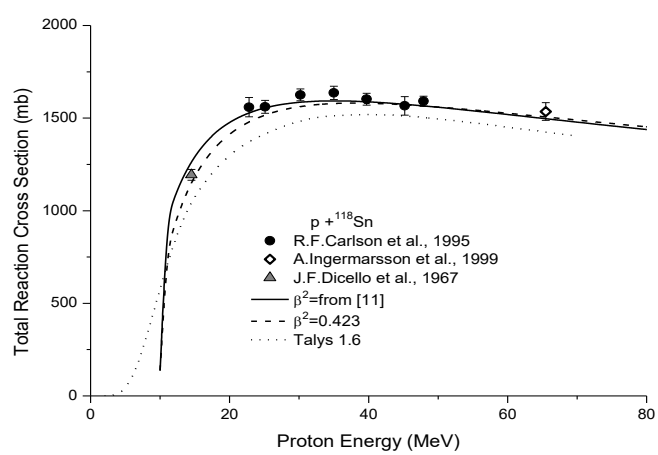

Figure 4. Calculated excitation function curves and experimental values for ${ }^{118} \mathrm{Sn}$ target nucleus. Experimental values were taken from Ref. [2]

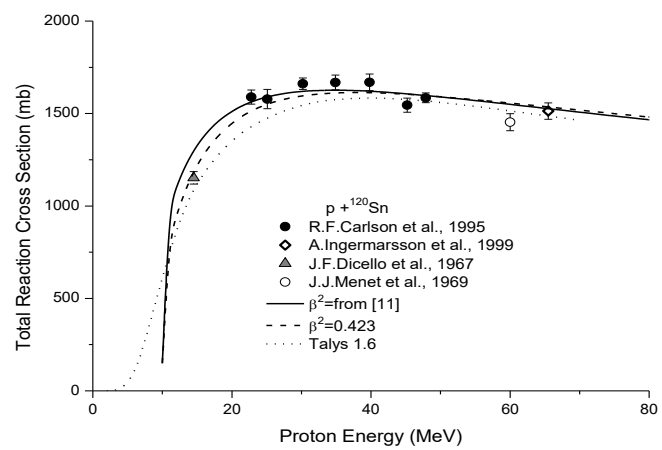

Figure 5. Calculated excitation function curves and experimental values for ${ }^{120} \mathrm{Sn}$ target nucleus. Experimental values were taken from Ref. [2]

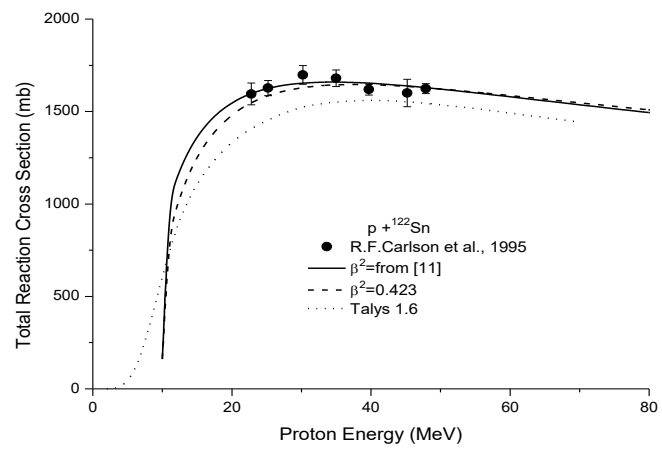

Figure 6. Calculated excitation function curves and experimental values for ${ }^{122} \mathrm{Sn}$ target nucleus. Experimental values were taken from Ref. [2] 


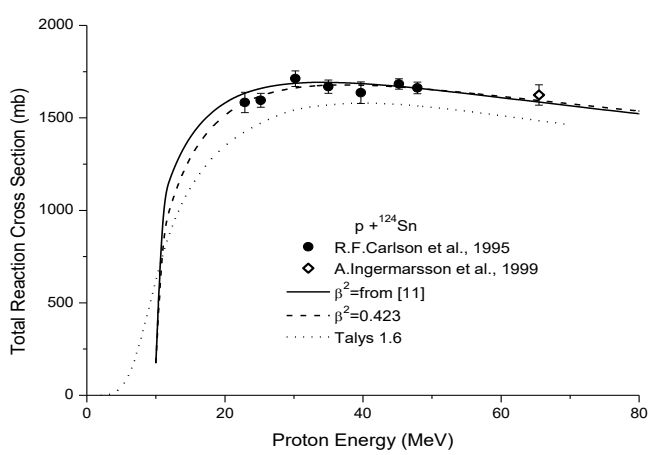

Figure 7. Calculated excitation function curves and experimental values for ${ }^{124} \mathrm{Sn}$ target nucleus. Experimental values were taken from Ref. [2]

As can be seen from the figures, in general, calculated results are in good harmony with the its experimental equivalents. While, cross section gets maximum value at about $35 \mathrm{MeV}$ energies, calculated $\beta_{N N}^{2}$ coefficient is equal to maximum values at $1.32 \mathrm{fm}^{2}$ about $10-12 \mathrm{MeV}$. $\beta_{N N}^{2}$ coefficient values have a decrease up to about $0.32 \mathrm{fm}^{2}$ with the increasing of proton incident energy up to $80 \mathrm{MeV}$. Fixed value at 0.423 calculations differs from the calculated value at the energy range of 10 and $20 \mathrm{MeV}$ energies. The excitation function that obtained from coefficient $c_{1}$ at 0.36 values are in best agreement with the experimental data in Figures. 4-7. When $c_{1}$ quantity valuated at proposed value 0.4 , calculations are above the experimental values. Calculated by the program TALYS, are demonstrated for comparing in Figures 1-7. All TALYS calculations also in good agreement. The calculated curve reasonably passes through the experimental data.

\section{Discussion and Conclusion}

In this study, proton induced total reaction cross section calculations have been determined for Sn target isotopes by analytical expression at Equation (2.1) (2.2) and by optical model parameterisations using TALYS code. Coefficients that are inside of analytical expression Equation (2.1) (2.2) have been compared owing to excitation function results in figures. Agreeable coefficient values have been defined. Also, a comparison was made between modelled calculati- ons and its experimental matching up to $80 \mathrm{MeV}$ proton incident energy. Good overall agreement was obtained. Alvi 's proton-nucleus total reaction cross section relation and TALYS code are applicable in cross section calculations for these isotopes. It is expected that this study contributes to concerned literature such as cross section studies and nuclear database.

\section{References}

1. Alvi, M.A, Study of proton total reaction cross section using a Helm model nuclear form factor, Nuclear Physics A, 2007, 789, 73-81.

2. EXFOR/CSISRS (Experimental Nuclear Reaction Data) Database Version of June 13, 2016 Brookhaven National Laboratory, National Nuclear Data Center), http://www.nndc.bnl.gov/exfor/exfor00.htm (accessed 21.06.2016).

3. Glauber, R.J, In: Lectures in Theoretical Physics, vol. 1. Interscience, New York, 1959; pp 315.

4. Büyükuslu, H, Kaplan, A, Aydın, A, Tel, E, Yıldırım, G, Calculation of Proton Total Reaction Cross Sections for Some Target Nuclei in Incident Energy Range of 10-600 MeV, Physics of Atomic Nuclei, 2010, 73 (10), 1700-1706.

5. Koning, A.J, Hilaire, S, Duijvestijn, M.C, TALYS-1.0, Proceedings of the International Conference on Nuclear Data for Science and Technology, 2007, April 22-27, pp 211-214.

6. Koning, A, Hilaire, S, Goriely, S, Talys-1.6, User Manual, Nuclear Research and Consultancy Group (NRG), Petten, The Netherlands, 2013, December 23.

7. Koning, A.J, Delaroche, J.P, Local and global nucleon optical models from $1 \mathrm{keV}$ to $200 \mathrm{MeV}$, Nuclear Physics A, 2003, 713, 231-310.

8. Friedrich, J, Voegler, N, The salient features of charge density distributions of medium and heavy even-even nuclei determined from a systematic analysis of elastic electron scattering form factors, Nuclear Physics A, 1982, 373, 192-224.

9. Charagi, S.K, Gupta, S.K, Coulomb-modified Glauber model description of heavy-ion reaction cross sections, Physical Review C, 1990, $41,1610-1618$

10. Ahmad, I, Abdulmomen, M.A, Al-Khattabi, L.A, Alpha-nucleus total reaction cross section in the rigid projectile model using microscopic $\mathrm{n}-\alpha$ amplitude, International Journal of Modern Physics E, 2001, 10, 43-53.

11. Horiuchi, W, Suzuki, Y, Abu-Ibrahim, B, Kohama, A, Systematic analysis of reaction cross sections of carbon isotopes, Physical Review $C, 2007,75,044607$. 